\title{
VACCINATION DURING PREGNANCY
}

\section{WOMEN IN GREECE ARE UNAWARE AND EXPRESS CONCERNS FOR SAFETY}

$\underline{\text { I. Kopsidas }^{1}}{ }^{2}$, F.E. Dasoula ${ }^{3}$, C. Mystakelis ${ }^{3}$, E. Kourkouni ${ }^{1}$, D.N. Maritsi ${ }^{3}$, G. Vartzelis ${ }^{3}$, M. Tsolia², N. Spyridis².

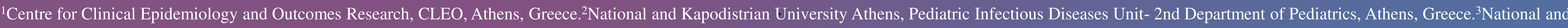
Kapodistrian University Athens, 2nd Department of Pediatrics- "P. \& A. Kyriakou" Children's Hospital, Athens, Greece.

\section{BACKGROUND}

Flu and pertussis vaccines are recommended in pregnancy as an important measure for preventing infection to mothers and newborns. Although there is good evidence on safety of both vaccines, uptake is very low in Greece.

We aimed to describe the knowledge and attitude of mothers in Greece on vaccination during pregnancy and identify barriers and facilitators that could be used to increase uptake.

\section{METHODS}

This was a cross-sectional survey that was conducted with an anonymous questionnaire of 34 items on a person-to-person interview with mothers of children presented at the emergency department or hospitalized at a tertiary children's hospital in Athens, Greece. Data collected included demographics, questions on knowledge and perceptions, and vaccination status during the last pregnancy.

\section{RESULTS}

154 mothers of a median age of 33 years (IQR:28-37) responded to the questionnaire and $47.7 \%$ had a higher education.

$125(81.2 \%)$ have given birth after $1 / 1 / 2013$ and

$14(11.2 \%)$ were vaccinated for flu and

$1(0.08 \%)$ for pertussis

Most frequent reasons given for non-vaccination for flu and pertussis respectively, were

a) unawareness $(54.4 \%, 84.8 \%)$

b) Safety $(34.4 \%, 8.8 \%)$

c) their gynecologist advised them not to $(13.6 \%, 8 \%)$

Even though the majority $(92.8 \%)$ believes that vaccines are beneficial, only $36.8 \%$ believe that flu vaccination is safe during pregnancy and $18.4 \%$ for pertussis with higher rates among mothers who gave birth after 2013(Table1)

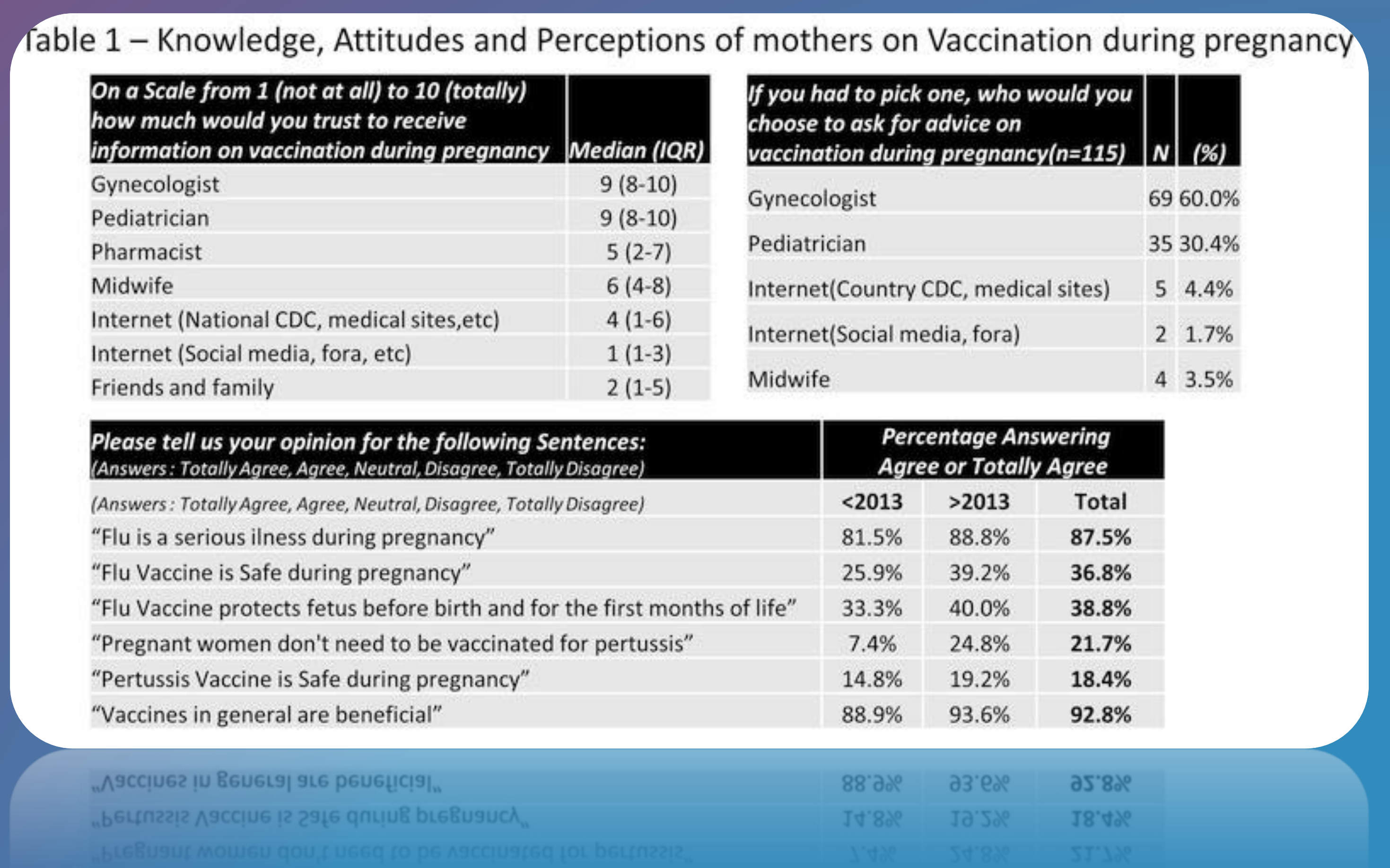

\section{CONCLUSIONS}

We detected very low rates of vaccination for flu or pertussis during pregnancy in Greece with high percentages of mothers admitting unawareness and expressing concerns on safety.

The majority would trust their gynecologist for information. Education and incorporating vaccination during routine pregnancy appointments are necessary actions to improve uptake. 